\title{
4 Zentralisierung der Notfallversorgung: Wie gewährleisten wir die Erreichbarkeit?
}

Boris Augurzky, Andreas Beivers und Alexander Haering

\subsection{Ausgangslage}

Eine jederzeit verfügbare medizinische Notfallversorgung von Patienten mit akuten Gesundheitsstörungen ist eine essenzielle Leistung für die Bevölkerung eines fortschrittlichen Industrielandes. Jedoch weist aktuell die Notfallversorgung in Deutschland vielfältige Probleme und Ineffizienzen auf. Eine Reform der Notfallversorgung steht daher im gesundheitspolitischen Fokus. Die Organisation einer erreichbaren Notfallversorgung ist Aufgabe ambulanter und stationärer Leistungserbringer, unterstützt durch den Rettungsdienst. Den Patienten stehen dabei im subjektiven Notfall, nach eigenem Ermessen, alle drei beteiligten Bereiche zu Verfügung.

Die Sicherstellung eines flächendeckenden Bereitschaftsdienstes obliegt in Deutschland den 17 regionalen Kassenärztlichen Vereinigungen (KVen). Jedoch befindet sich dieser vertragsärztliche Bereitschaftsdienst in einem Wandel: In der Vergangenheit wurde er vorwiegend durch Angebote der zugelassenen Ärzte in ihrer eigenen Praxis oder durch Hausbesuche sichergestellt. In jüngster Zeit wird er jedoch zunehmend durch feste Anlaufpraxen in Ergänzung durch Fahrdienste, koordiniert durch den kassenärztlichen Notdienst - bundesweit erreichbar unter der Telefonnummer 116117 - organisiert.

Neben dem Bereitschafsdienst durch die KVen sind auch zugelassenen Krankenhäuser (nach $\$ 108$ SGB V) zur Teilnahme an der Notfallversorgung verpflichtet. Krankenhäuser können im Notfall alle dafür vorgesehenen EBM-Leistungen mit 


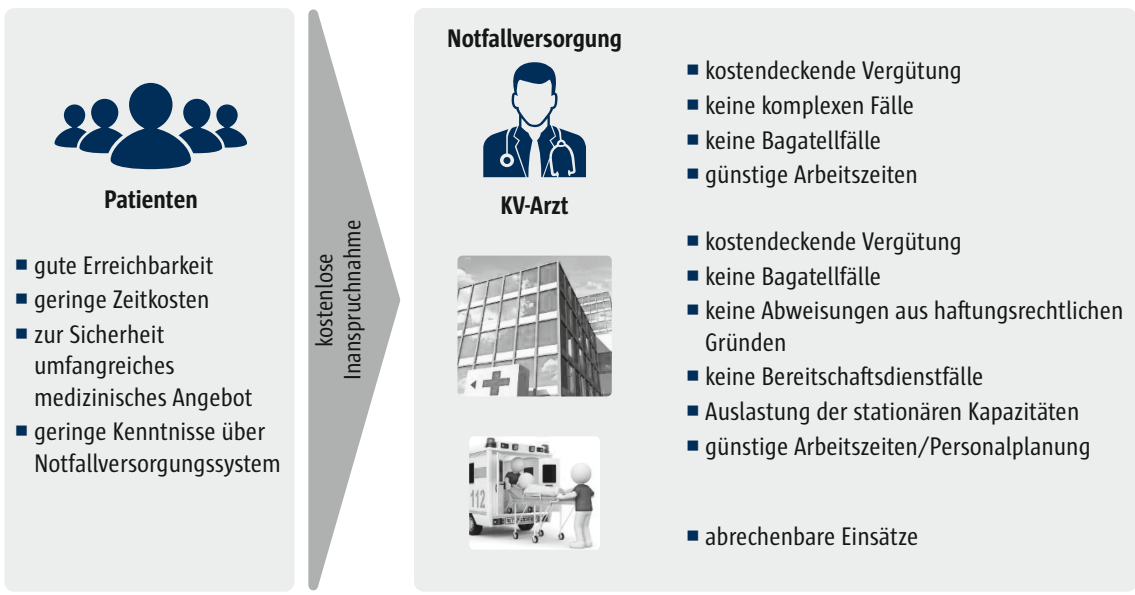

Abb. 1 Einzelne Akteure mit divergierenden Interessen (RWI 2018)

der regionalen KV abrechnen. Einer gesonderten Ausnahmeregelung bedarf es momentan noch nicht.

Die Notfallversorgung in Deutschland beruht im Wesentlichen auf gewachsenen historischen Strukturen und zeigt regionale Unterschiede aufgrund der föderalen Organisation durch die einzelnen Bundesländer (Niehues 2012; Augurzky et al. 2013). Weiter kann in der deutschen Notfallversorgung beobachtet werden, dass verschiedene Akteure - ambulant tätige Ärzte, der stationäre Sektor sowie das Rettungswesen - teilweise verschiedene Ziele verfolgen. Dies führt in einigen Fällen zu Spannungsverhältnissen, welche auf den verschiedenen Interessen dieser Akteure beruhen. Abbildung 1 stellt eine kurze Übersicht zu den einzelnen Zielen der Akteure dar. Hier fällt vor allem das vielschichtige Spannungsverhältnis zwischen ambulanten (KV-Arzt) sowie stationärem (zweite Abbildung von oben) Sektor ins Auge. Jedoch fällt bei genauerer Betrachtung auf, dass viele dieser Punkte durch eine strukturierte Ausgestaltung, mit klaren Regeln und Zuständigkeiten, in Einklang gebracht werden können. So sind z.B. die Punkte „keine komplexen Fälle“ der KV-Ärzte und „keine Bereitschaftsdienstfälle“ des stationären Sektors ohne Abstriche in Einklang zu bringen. Hier bedarf es einer effizienten Steuerung der Patienten, sodass sie immer in den ihrem Risiko entsprechendem Versorgungsstrang gelenkt werden.

Weiter kann beobachtet werden, dass teilweise starke regionale Unterschiede in den verfügbaren Strukturen der Notfallversorgung vorliegen. So zeichnet sich die Notfallversorgung einerseits dadurch aus, dass eine große Anzahl an Gebieten in Deutschland teilweise überversorgt ist und auf der anderen Seite Regionen eher die Tendenz zur Unterversorgung aufweisen. Zudem stellt sich selbst in objektiv überversorgten Regionen und Städten das subjektive Gefühl der Unterversorgung ein (Augurzky u. Beivers 2015): So waren im Jahr 2015 
knapp 50\% der stationär aufgenommenen Krankenhauspatienten sogenannte Notfälle. In den Notaufnahmen liegt der Anteil der stationär aufzunehmenden Notfallpatienten zwischen 30 und $55 \%$. Dies zeigt, wie intensiv die deutschen Krankenhäuser in der Notfallversorgung beansprucht werden. In der Konsequenz kommt es immer wieder zu überfüllten und überlasteten Notfallaufnahmen.

Die hier geschilderten Probleme wurden im Bereich der ambulanten wie auch stationären Notfallversorgung von der Politik erkannt. So sah bereits das Krankenhausstrukturgesetz (KHSC) Neuregelungen v.a. für den Bereich der stationären Notfallversorgung vor, wie beispielsweise ein abgestuftes System mit konkreten Struktur- und Prozessvorgaben für teilnehmende Kliniken (Augurzky et al. 2017). Hierbei wurde ausdrücklich festgestellt, dass nicht jedes somatische Krankenhaus an der Notfallversorgung teilnehmen sollte. Dies ist aus versorgungstechnischen wie auch wirtschaftlichen Gesichtspunkten zu begrüßen.

Weiter legt das KHSG fest, die Patientensteuerung zwischen ambulantem und stationärem Sektor in der Notfallversorgung zu verbessern. Es sieht dabei vor, dass zur ambulanten Versorgung von Notfällen mit niedrigem Risiko vertragsärztliche Notdienstpraxen (sogenannte Portalpraxen) in unmittelbarer Nähe oder direkt im teilnehmenden Krankenhaus eingerichtet werden sollen. So sollen die Notfallambulanzen der Kliniken direkt in den Notdienst eingebunden werden. Ziel ist es dabei, einen effektiven Steuerungsmechanismus zu etablieren, welcher vor Eintritt in das System den individuellen Versorgungsbedarf der Patienten ermittelt und auf dieser Basis den Patienten in eine für ihn adäquate Versorgung leitet.

Der hier vorliegende Beitrag setzt sich mit der Frage auseinander, wie die regionale Neuordnung der Notfallversorgung in Deutschland realisiert werden kann. Hierfür wird analysiert, wie viele Krankenhäuser an der Notfallversorgung teilnehmen sollten. Dabei wird aufgezeigt, dass, ähnlich wie im KHSC festgestellt, nicht jedes somatische Krankenhaus in Deutschland an der Notfallversorgung teilnehmen muss, um eine flächendeckende Versorgung sicherzustellen. Hierfür stellt der Beitrag kurz die für die hier aufgestellte Fragestellung wesentlichen Punkte der vom RWI - Leibniz-Institut für Wirtschaftsforschung durchgeführten Studie zum Thema „Notfallversorgung in Deutschland. Projektbericht im Auftrag der Kassenärztlichen Bundesvereinigung “ dar. Ziel des Projektes war es, die Anzahl an Krankenhäuser zu bestimmen, welche um eine Bereitschaftsdienstpraxis, betrieben von ambulant praktizierenden Ärzten der lokalen KV, ergänzt werden sollten. 


\subsection{Räumliche Verteilung von Notfallzentren unter Gewährleistung der Erreichbarkeit: Ergebnisse des KBV-Gutachtens}

\subsubsection{Datengrundlage: Prävalenzraten und Inanspruchnahme}

Neben der Bevölkerungsverteilung bilden die auf den Prävalenzraten basierenden erwarteten Fallzahlen auf Kreisebene die Grundlage für die Simulation des Bedarfs an Notfallzentren in Deutschland. Zur Ableitung der regionalen Fallzahlen wurden die vom Zentralinstitut für die kassenärztliche Versorgung (Zi)

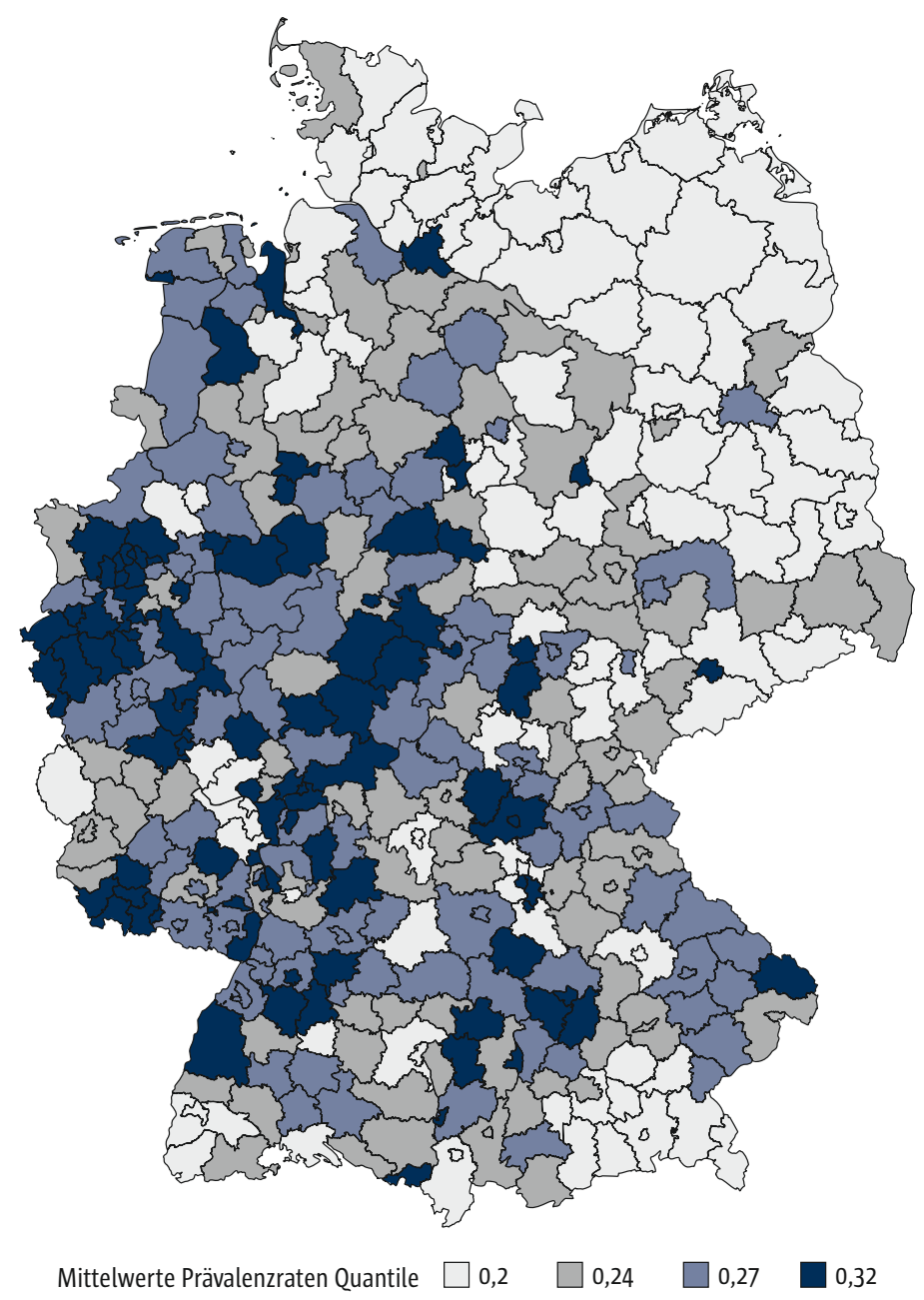

Abb. 2 EBM 1.2 Fälle pro Einwohner und Jahr auf Kreisebene (2015) in Quantilen (RWI 2018). Anmerkung: Zellen mit weniger als 30 Fällen beinhalten im Durchschnitt 3.596 Fälle; beachtet werden auch Fälle, bei denen das Geschlecht nicht erfasst wurde; Anzahl Fälle wurde um PKV-Versicherte approximativ erweitert; für Bremen wurde die Prävalenzrate von Niedersachsen angenommen. 
bereitgestellten Abrechnungsdaten der KVen für das Jahr 2015 herangezogen. Diese Daten beinhalten alle Behandlungsfälle auf Kreisebene, welche nach EBM 1.2 als Notfall (administrative Notfalldefinition) abgerechnet wurden, eingeteilt in Altersklassen differenziert nach Geschlecht. Für den privatärztlichen Notdienst wurden Fälle von privat versicherten Personen approximativ nach Anteil der privat versicherten Personen je Bundesland aufgeschlagen.

Abbildung 2 stellt die berechneten Prävalenzraten, eingeteilt in die Kreise Deutschlands, dar. Bei genauer Betrachtung fällt auf, dass innerhalb eines Bundeslands die Prävalenzrate teilweise stark variiert. Zudem befindet sich ein Croßteil der Kreise mit der niedrigsten Rate in den neuen Bundesländern und der Croßteil der Kreise mit den höchsten Prävalenzraten in Ballungsgebieten, z.B. in der Rhein-Ruhr-Region. Die beobachteten Unterschiede können aufgrund des Zusammenhangs von ambulanter Versorgung und stationärer Aufnahme in Abhängigkeit von freien Kapazitäten der Krankenhausstationen erklärt werden. Somit hat die generelle Auslastung der Krankenhäuser in den verschiedenen Regionen Deutschlands einen Einfluss auf die Wahrscheinlichkeit, einen Fall ambulant zu versorgen oder stationär aufzunehmen (Kopetsch 2006).

Eine Aufteilung der Prävalenzrate nach Alter und Geschlecht ist in Abbildung 3 dargestellt. Diese bildet (bereinigt um regional bedingte Verzerrungen) die Grundlage für die Simulationen (Augurzky et al. 2018). Begründen lässt sich die Verwendung dieser bundeseinheitlichen Rate mit der vorliegenden Endogenität (s.o.). Eine Nutzung der kleinräumigen Raten auf Kreisebene würde somit die Ergebnisse der Simulation verzerren (vgl. Augurzky et al. 2018, S. 34).

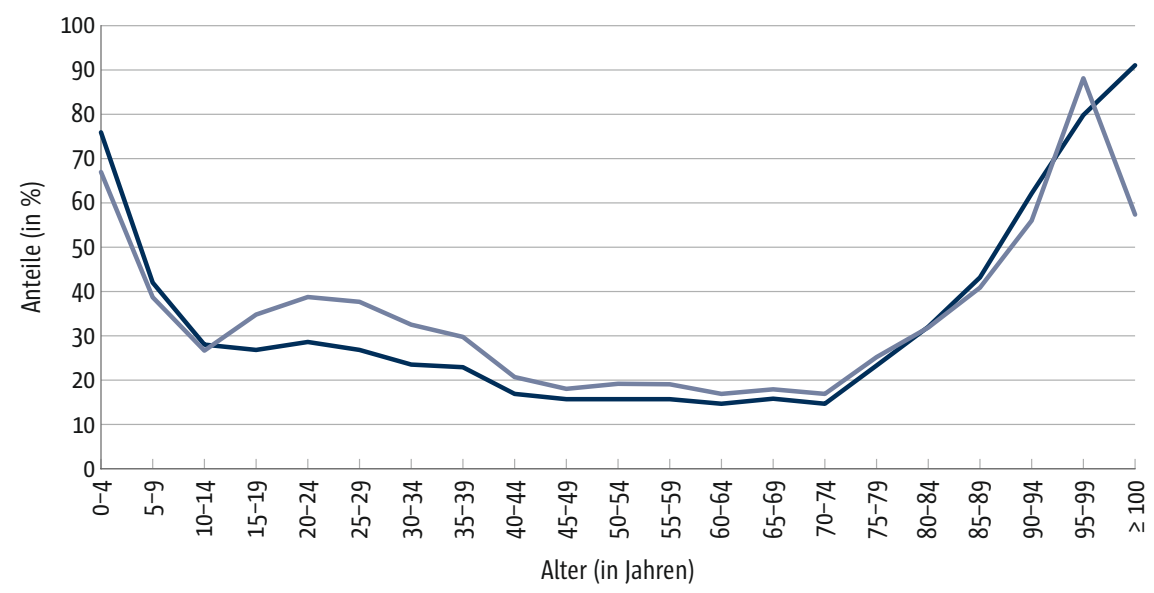

— Prävalenzrate Männer ——Prävalenzrate Frauen

Abb. 3 EBM 1.2 Fälle pro Einwohner und Jahr nach Alter und Geschlecht. Quelle: RWI (2018). Anmerkung: Zellen mit weniger als 30 Fällen beinhalten im Durchschnitt 3.596 Fälle; beachtet werden nur Fälle, bei denen das Geschlecht erfasst wurde; Anzahl Fälle wurde um PKV-Versicherte approximativ erweitert; für Bremen wurde die Prävalenzrate von Niedersachsen angenommen. 
Für eine genaue Simulation der benötigten Notfallzentren ist weiter die Verteilung der Fälle über den Tagesverlauf an Samstagen, Sonntagen und Feiertagen von Relevanz: Im derzeit vorherrschenden Öffnungszeitenmodell findet das höchste Patientenaufkommen in den Bereitschaftspraxen am Wochenende vormittags statt, während Fälle in der tiefen Nacht deutlich weniger häufig zu verzeichnen sind (DKI Krankenhausbarometer 2017). Aufbauend auf dieser Beobachtung lassen sich im Rahmen der Simulation die zu erwartenden Fallzahlen für unterschiedliche Öffnungszeitmodelle (momentane Öffnungszeiten des Bereitschaftsdiensts sowie eine Öffnung 24/7) für Notfallzentren ableiten.

\subsubsection{Simulation der optimalen Anzahl an Notfallzentren}

Die Standortoptimierungen, durchgeführt im Rahmen der RWI-Studie, basieren auf Rastereinheiten von $1 \mathrm{~km}^{2}$. Durch eben diese Raster ist die zwingend notwendige kleinräumige Betrachtung im Rahmen der Simulation möglich. Die etwa 360.ooo Quadranten für ganz Deutschland halten, gerade auch im ländlichen Bereich, genauso feingliedrige Informationen bereit wie in dicht besiedelten Gebieten. In der Analyse wurden unbewohnte Rastereinheiten ausgeschlossen. Die Gesamtzahl der für die Analyse relevanten bewohnten Raster beläuft sich nach Bereinigung auf 200.ooo. Für diese Raster liegen sozioökonomische Charakteristika der Bevölkerung vor (vgl. RWI GEO GRID in Budde u. Eilers 2014), welche für die Simulation von entscheidender Bedeutung sind: Es handelt sich um Informationen zur Einwohnerzahl, unterteilt in jeweils 17 Gruppen für beide Geschlechter. Mithilfe dieser Daten und in Verbindung mit den oben beschriebenen Prävalenzraten nach Geschlecht und Alter, können die hypothetisch zu erwartenden Fallzahlen auf Rasterebene bestimmt werden, welche die Versorger abdecken müssen.

Die Berechnung der Fahrzeiten zwischen den Rastern wird auf Basis von OpenStreetMap-Daten durchgeführt (vgl. Ramm u. Topf 2010). Für die Simulation der Erreichbarkeit müssen vorab Prämissen und Eckwerte der Versorgung definiert werden. Dabei wird eine maximale Entfernung von 30 Minuten Fahrzeit zum nächstliegenden Versorger festgelegt. Es werden durchschnittlich Geschwindigkeiten für 15 verschiedene Straßentypen zugrunde gelegt. Eine Beachtung des aktuellen Verkehrsaufkommens wird dabei jedoch nicht berücksichtigt, um eine etwaige kurzfristige Verzerrung durch z.B. Bauaktivitäten zu verhindern. Weiter wird festgelegt, dass nur solche Raster als Standorte infrage kommen, welche ein Mindestmaß an Infrastruktur gewähren. Es wird somit eine geglättete Einwohnerdichte von mindestens 150 Einwohnern als Voraussetzung für den Standort festgelegt.

Unter der Bedingung einer flächendeckenden Erreichbarkeit von Notfallzentren werden in der RWI-Studie zwei unterschiedliche Simulationsansätze zur Ermittlung der notwendigen Mindestanzahl solcher Notfallzentren unterschieden. Zum einen „Grüne Wiese“ und zum anderem „Reale Standorte“. 
Tab. 1 Versorgungslage verbliebene Bevölkerung (RWI, eigene Berechnungen). Annahme: Es wurden potenzielle Versorger ausgeschlossen, welche weniger als $1 \%$ der Bevölkerung oder weniger als 15 Raster versorgen.

\begin{tabular}{|c|c|c|}
\hline Maximale Fahrzeit zum Versorger (in Minuten) & Bevölkerung & Anteil \\
\hline 30 & 79.957.613 & $99,00 \%$ \\
\hline 35 & 80.579 .886 & $99,77 \%$ \\
\hline 40 & 80.663 .493 & $99,87 \%$ \\
\hline 45 & 80.675 .789 & $99,89 \%$ \\
\hline 60 & 80.679 .045 & $99,89 \%$ \\
\hline
\end{tabular}

Im ersten Ansatz werden die benötigten Versorger unter Berücksichtigung der oben erwähnten Prämissen frei in Deutschland verteilt. Es werden 337 Zentren für eine Versorgung von 99,0\% der Bevölkerung benötigt.

Hier werden etwa 208.ooo hypothetische Fällen pro Jahr nicht innerhalb von 30 Minuten versorgt. Bei genauer Betrachtung der Ergebnisse fällt jedoch auf, dass innerhalb von 35 Minuten schon 99,8\% der Bevölkerung versorgt sind (s. Tab. 1). Bei einer Ausweitung der maximalen Fahrzeit auf 40 Minuten sind es bereits 99,9\% der Einwohner Deutschlands. Der Bevölkerungsteil, welcher von den hier abgeleiteten Versorgern nicht innerhalb von 6o Minuten versorgt wird, lebt fast ausschließlich auf Inseln. Eine Beachtung innerhalb der Regelversorgung ist somit nur schwer möglich. Jedoch können hier lokale Lösungen gefunden werden, welche auf die regionalen Gegebenheiten angepasst sind. Denkbar wären dabei z.B. ein Ausbau der Luftrettung oder eine Ausweitung des Fahrdienstes.

Im zweiten Ansatz wird bei der Verteilung der Zentren die in Deutschland vorhandene Krankenhausstruktur explizit berücksichtigt. Dabei wird abgeleitet, wie viele Versorgerstandorte benötigt werden, wenn die Versorger nicht, wie im „Grüne-Wiese“-Modell, frei und somit optimal verortet werden, sondern potenzielle Notfallversorger nur an den bestehenden (und bisher an der Notfallversorgung teilnehmenden) 1.456 somatischen Krankenhäusern lokalisiert werden. Das Resultat ist in Abbildung 4 dargestellt. In 59 Fällen befinden sich zwei oder mehr Krankenhäuser so dicht beieinander, dass sie in das gleiche Raster fallen. Somit kommt es auf Rasterebene zu 1.393 „effektiven“ Standorten. Hier wird eine Erreichbarkeit von maximal zo Minuten Fahrzeit für 99,6\% der Bevölkerung durch 736 Notfallzentren erreicht.

Die oben dargestellten Ergebnisse machen deutlich, dass eine flächendeckende Notfallversorgung mit weniger Standorten als im Status quo möglich wäre. Dies erscheint aus wirtschaftlichen und versorgungstechnischen Gesichtspunkten sinnvoll. Jedoch stellen die 337 Standorte auf Grundlage des Ansatzes „Grüne Wiese“ eher einen hypothetischen Wert dar, da bei einer geografisch völlig freien Verortung der Notfallversorger, die im Hintergrund ggf. nötige 

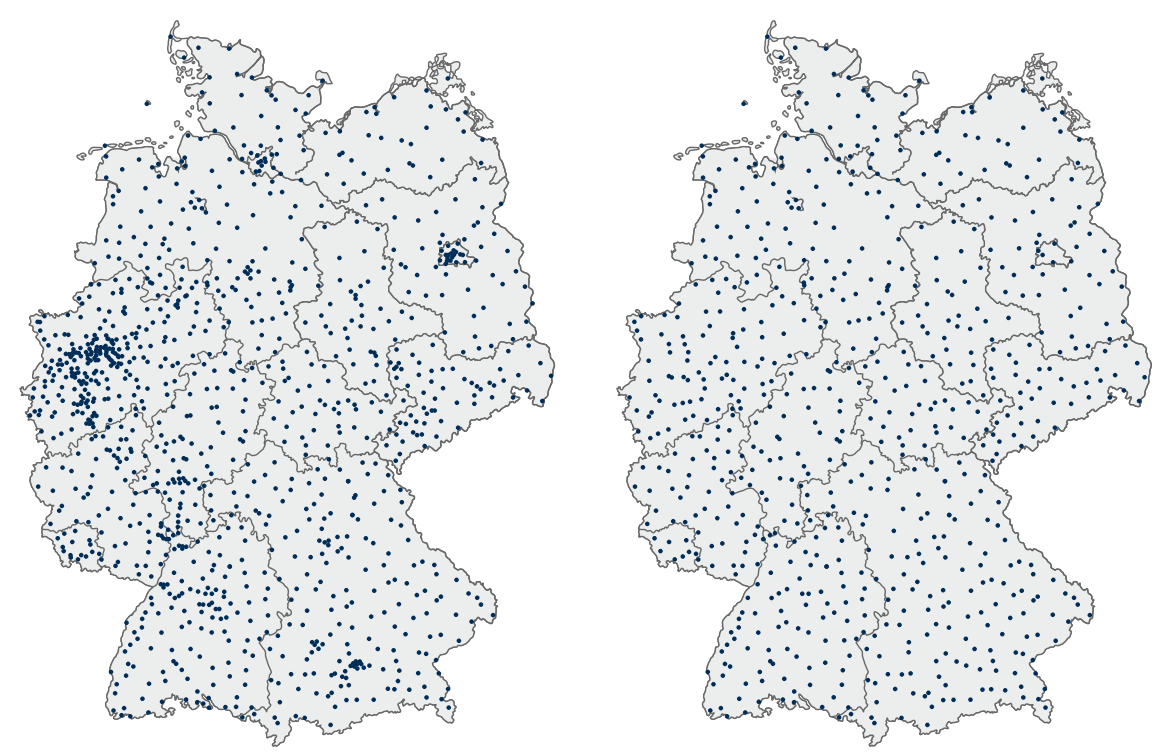

Abb. 4 Lage der tatsächlichen und der notwendigen Krankenhausstandorte mit Notfallversorgung. Links: alle 1.393 momentanen (effektiven) Standorte; rechts: 736 notwendige Standorte für eine 30-Min.-Versorgung (RWI 2018). Anmerkung: notwendige Versorger ermittelt via greedy set cover-Algorithmus auf Basis der momentanen Standorte

Krankenhausinfrastruktur nicht immer vorhanden sein kann. Jedoch zeigt das Ergebnis der Simulation „Reale Standorte“, dass eine Konzentration der Notfallversorgung auf 736 Standorte möglich wäre. Die flächendeckende Erreichbarkeit für die Bevölkerung wäre dabei in keiner Weise gefährdet.

\subsubsection{Maximalgröße und Wirtschaftlichkeit der Notfallzentren}

Es ist theoretisch möglich, dass beispielsweise in Ballungsgebieten ein einziges sehr großes Notfallzentrum sehr viele Patienten wohnortnah versorgt. In der Praxis ist es jedoch sinnvoll, solche „Megazentren“ aufzusplitten. Ziel wäre dabei eine überschaubare, aber weiterhin noch wirtschaftliche Größe der Zentren. Einrichtungen, welche mehr als 170.000 Fälle pro Jahr versorgen, können somit entsprechend aufgeteilt werden. Dies würde im Ansatz „Reale Standorte "zu bundesweit 751 Standorten für eine flächendeckende Versorgung bei einer 24/7-Öffnung führen. Eine maximal sinnvolle Kapazität von 170.0oo Fällen pro Jahr ergibt sich dabei aus der im Hintergrund zur Verfügung stehenden angebundenen Krankenhaus-Infrastruktur. In einem zweiten Schritt werden, auf Basis der oben dargestellten Simulation „Reale Standorte“, die Zentren auf ihre Wirtschaftlichkeit geprüft. Abhängig vom zugrunde liegenden Öffnungszeitenmodell (derzeitige eingeschränkte Öffnungszeiten oder „24/7-Öffnung“), ist der Betrieb der Notfallzentren mit hohen personellen Ressourcen verbunden. Dies kann, je nach Region, zu teilweise nicht unerheb- 
lichen wirtschaftlichen Defiziten führen. Bei Einhaltung der derzeitigen Öffnungszeiten werden 738 Standorte für eine flächendeckende Versorgung benötigt. Basierend auf der Vergütungssituation im Jahr 2018 für den Bereitschaftsdienst würden hierbei 527 Standorte ein Defizit erwirtschaften. In der Summe würde sich ein Defizit über alle Standorte auf insgesamt rund 130 Mio. Euro jährlich summieren.

Bei einer ganztägigen Öffnung über das gesamte Jahr hinweg wären 751 Standorte nötig, davon 581 defizitär mit einem Gesamtdefizit von rund 400 Mio. Euro. Pro Notfallzentrum würde der durchschnittliche Verlust eines der 581 defizitären Zentren rund o,7 Mio. $€$ p.a. betragen. Dabei ist zu beachten, dass diesen Zahlen nur modellhafte Berechnungen zugrunde liegen. In der Versorgungsrealität sollte immer im Einzelfall geprüft werden, welches Öffnungszeitenmodell bzw. welche personelle Ausstattung an einem Standort nötig und wirtschaftlich sinnvoll ist. Als bewiesen kann jedoch gelten, dass wesentlich weniger Standorte für die Notfallversorgung als im Status quo ausreichend sind, um eine effiziente und flächendeckende Versorgung sicherzustellen.

\subsection{Fazit}

Im Rahmen einer Simulationsanalyse wurde im hier vorliegenden Beitrag die Mindestanzahl an Notdienstzentren abgeleitet. Bei der hier aufgezeigten Verteilung der Zentren wurde die in Deutschland vorhandene Krankenhausstruktur explizit berücksichtigt. Somit können potenzielle Zentren nur an bestehenden - und an der Notfallversorgung teilnehmenden - Krankenhäusern lokalisiert werden. Hier wird das Erreichbarkeitsniveau von maximal 30 Minuten Fahrzeit für 99,6\% der Bevölkerung mit 736 Notfallzentren erreicht. Diese Zahl liegt weit unter den 1.456 somatischen Krankenhäusern, welche im Jahr 2014 offiziell an der Notfallversorgung teilnahmen, auch wenn verschiedene Öffnungszeitenmodelle sowie eine Aufteilung von sehr großen Zentren auf mehrere Standorte unterstellt wird.

Dies macht deutlich, dass eine flächendeckende Notfallversorgung in Deutschland mit deutlich weniger Standorten möglich wäre und aus wirtschaftlichen und versorgungstechnischen Gesichtspunkten sinnvoll erscheint. Bei einer flächendeckenden Einführung von z.B. Portalpraxen an bestehenden Krankenhäusern ist es also nicht nötig, dass alle momentan an der Notfallversorgung teilnehmenden Krankenhäuser diesen Status beibehalten bzw. durch eine KV-Notfallpraxis ergänzt werden müssen. Zudem können durch eine Bündelung der Notfallversorgung auf versorgungstechnisch erforderliche Standorte Vorteile in der Versorgung durch eine Konzentration der Leistungserbringung erzielt werden.

Ein Nachteil in der oben dargestellten Simulation könnte darin bestehen, dass im Rahmen der Berechnungen der Fahrzeiten nicht reale Verkehrssituationen vor Ort beachtet werden konnten. Hier wurde auf standardisierte Fahrzeiten für 15 verschiedene Straßentypen zurückgegriffen. So kann es in der Realität 
zu leichten Abweichungen in der tatsächlichen Erreichbarkeit kommen, gerade zum Beispiel in Ballungsgebieten wie Großstädten oder dem Ruhrgebiet. Auch wurde bei der Verteilung der potenziellen Zentren keine maximale Kapazität pro Versorger (z.B. maximale Anzahl an Patienten pro Tag) unterstellt. Hier könnte es ggf. zu einzelnen marginalen Abweichungen kommen, wenn nicht wie hier „Megazentren“ nachträglich aufgeteilt werden (vgl. Kap. 4.2.3).

Die oben dargestellten Grenzen der Simulation können jedoch vernachlässigt werden, wenn die Ausgestaltung der lokalen Verteilung der Standorte auf regionaler Ebene erfolgt. Hier kann vor Ort und unter Berücksichtigung der regionalen Gegebenheiten entschieden werden, ob und an welche Krankenhäuser eine KV-Notfallpraxis angedockt wird. Zudem kann geprüft werden ob eine ggf. neu geschaffene, freistehende Praxis nötig und sinnvoll ist. In diesem Prozess sollten die lokalen KVen und die Krankenhäuser in die Entscheidungsfindung integriert werden. Eine vertragliche Fixierung der Zuständigkeiten sowie der notwendigen finanziellen Rahmenbedingungen kann zudem helfen, potenzielle Interessenkonflikte und wirtschaftliche Ineffizienzen zu vermeiden.

Neben der hier dargestellten optimalen Verortung der an der Notfallversorgung teilnehmenden Notdienstpraxen können eine Verbesserung der Patientensteuerung sowie klare Strukturvorgaben und Zuständigkeiten der beteiligten Einrichtungen flankierend wirken. Durch einen Blick auf Gesundheitssysteme außerhalb Deutschlands z.B. in Dänemark, Großbritannien, der Schweiz oder auch in den Niederlanden können sinnvolle Konzepte zur verbesserten Patientensteuerung gefunden werden. Dabei fallen wesentliche Konzepte der Notfallversorgung besonders häufig auf und können wichtige Ideen für Deutschland liefern (Augurzky et al. 2018).

\section{Ideen für sinnvolle Notfallversorgungskonzepte}

- Auskunfts-, Beratungs- und Leitstellen, die dem Erstkontakt der Patienten dienen (Portalpraxen).

- Allgemeinmediziner, die die ambulante Versorgung der Patienten mit medizinischen Notfällen ohne hohes Risiko übernehmen.

- Rettungsdienste, die Patienten mit einem hohen Gesundheitsrisiko in die Notaufnahmen der Krankenhäuser transportieren.

- Notaufnahmen der Krankenhäuser, in denen Patienten mit risikoreichen und aufwendig zu diagnostizierenden und behandelnden Krankheiten durch spezifisch ausgebildete Notfallmediziner erstversorgt werden.

Für das Erreichen einer bedarfsgerechten und qualitativ hochwertigen Versorgung von Notfallpatienten ist somit, neben einer optimalen Verortung, die 
Koordination und Steuerung aller beteiligten Einrichtungen von entscheidender Bedeutung. Generell ist in vielen Industrieländern eine Tendenz zu beobachten, dass die haus- und allgemeinärztliche Versorgung von Notfallpatienten mit niedrigem Risiko räumlich an Krankenhäuser verlagert wird. Jedoch bleibt sie weiter Aufgabe der Allgemeinärzte. Eine zuverlässige Ersteinschätzung zur Patientensteuerung innerhalb eines Notfallzentrums kann mit einem validierten, anerkannten Triage-System helfen, die Patienten in die für sie relevante Ebene der Notfallversorgung zu führen. Auch sollte die Aufgabenteilung zwischen der ambulanten Notfallversorgung durch qualifizierte Ärzte in den Praxen und der Notfallversorgung durch Notfallmediziner im Krankenhaus für die Patienten und alle beteiligten Berufsgruppen transparent festgelegt werden.

\section{Take home messages}

- Die Aufgabenteilung zwischen ambulanter Notfallversorgung in KV-Praxen und Notfallversorgung im Krankenhaus sollte für alle Beteiligten transparent festgelegt werden.

- Notdienstpraxen und Notaufnahmen sollten verzahnt werden: feste räumliche Zuordnung (z.B. gemeinsamer Tresen), festgeschriebenes Service-Level und abgestimmte Öffnungszeiten.

- Eine flächendeckende Versorgung wäre theoretisch mit 337 Standorten möglich. Durch erforderliche Anbindung an Krankenhausstandorte sind jedoch 736 Notfallzentren notwendig.

\section{Literatur}

Augurzky B, Beivers A (2015) Rettung für die Notfallmedizin. Gesundheit und Gesellschaft 18(10): 19-23

Augurzky B, Beivers A, Breidenbach P, Budde R, Emde A, Haering A, Kaeding M, Roßbach-Wilk E, Straub N (2018) Notfallversorgung in Deutschland. Projektbericht im Auftrag der Kassenärztlichen Bundesvereinigung. RWI - Leibniz-Institut für Wirtschaftsforschung (Hrsg.) Projektberichte, Essen

Augurzky B, Beivers A, Dodt C (2013) Schnelle Hilfe zentralisieren. f\&w führen und wirtschaften im Krankenhaus. 30(04), 431-435

Augurzky B, Beivers A, Dodt C (2017) Handlungsbedarf trotz Krankenhausstrukturgesetz: Elf Thesen zur Reform der Notfallversorgung. RWI Position 68, 05/Mai 2017, RWI, Essen

Budde R, Eilers L (2014) Sozioökonomische Daten auf Rasterebene: Datenbeschreibung der microm-Rasterdaten. RWI Materialien Nr. 77, Essen

DKI (2017) Krankenhausbarometer - Umfrage 2017. Deutsches Krankenhausinstitut e.V., Düsseldorf

Kopetsch T (2006) Gilt Roemer's Law auch in Deutschland? Jahrbücher für Nationalökonomie und Statistik 226(6): 646-669

Niehues C (2012) Notfallversorgung in Deutschland. Analyse des Status quo und Empfehlungen für ein patientenorientiertes und effizientes Notfallmanagement. Kohlhammer Verlag, Stuttgart

Ramm F, Topf I (2010) OpenStreetMap: Die freie Weltkarte nutzen und mitgestalten. Lehmanns Media Köln 


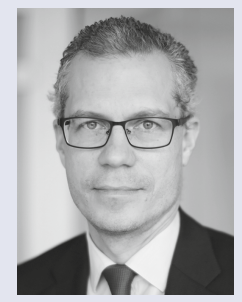

\section{Prof. Dr. Boris Augurzky}

Boris Augurzky ist seit 2003 Leiter des Bereichs "Gesundheit“ am RWI, seit 2007 Geschäftsführer der hcb GmbH, seit 2014 bei der Stiftung Münch, seit 2019 als Vorstandsvorsitzender und seit 2016 außerplanmäßiger Professor an der Universität Duisburg-Essen. Er ist Mitglied verschiedener Ausschüsse und Beiräte: der Deutschen Krebshilfe, des Vereins für Socialpolitik, des BMC, der IQM, des BARMER Institut für Gesundheitssystemforschung, des WIdO und des Rates für Gesundheit und Medizinethik des Bistums Essen.

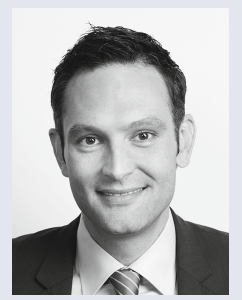

\section{Prof. Dr. Andreas Beivers}

Andreas Beivers ist seit 2010 Professor für Volkswirtschaftslehre und Gesundheitsökonomie an der Hochschule Fresenius in München; seit 2017 ist er assoziierter Wissenschaftler des Kompetenzbereichs „Gesundheit“, RWI Leibniz-Institut für Wirtschaftsforschung; seit 2015 Mitglied im Academic Board, University of Salzburg Business School; Lehrbeauftragter der TU München.

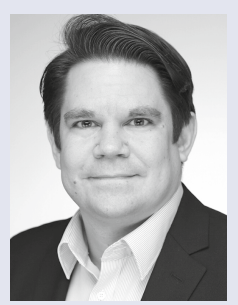

\section{Alexander Haering}

Alexander Haering schloss sich dem RWI im Januar 2017 an. Er studierte Betriebswirtschaftslehre - Gesundheitsökonomie und Management im Gesundheitswesen (M.Sc.) und Volkswirtschaftslehre (B.Sc.) an der Universität Duisburg-Essen. Momentan verfolgt er sein Promotionsvorhaben mit einem Schwerpunkt auf Verhaltensökonomik. Er ist des Weiteren assoziiert mit der Hochschule Fresenius. 